\title{
THREE SPECTROSCOPIC BINARIES IN THE BRIGHT STAR CATALOG SUPPLEMENT
}

\author{
C. D. Scarfe ${ }^{1,2}$ \\ Received November 28 2018; accepted February 282019
}

\begin{abstract}
This paper presents spectroscopic orbits of three binaries, HD 121212, HD 148434 and HD 148912, with evolved primaries and periods of order a year, found in a survey of late-type stars listed in the Supplement to the Yale Bright Star Catalog. All the orbits were determined from observations made with the DAO 1.2-m telescope and coudé spectrograph. Observations were obtained using the radial velocity spectrometer until it was decommissioned in 2004, and since then using a CCD detector and cross-correlating the spectra with those of standard stars.
\end{abstract}

\section{RESUMEN}

Se presentan las órbitas espectroscópicas de tres binarias (HD 121212, HD 148434 y HD 148912) con primarias evolucionadas y períodos de cerca de un año, encontradas en el relevamiento de estrellas tardías listado en el suplemento al Yale Bright Star Catalog. Las órbitas se determinaron a partir de observaciones hechas en el telescopio de $1.2 \mathrm{~m}$ del DAO con el espectrógrafo coudé. Las observaciones se obtuvieron con el espectrómetro para velocidades radiales antes de su cancelación en 2004, y a partir de entonces con un detector CCD y mediante la correlación cruzada de los espectros con estrellas estándar.

Key Words: binaries:spectroscopic

\section{INTRODUCTION}

About thirty years ago the author began a program of observations of the radial velocities of latetype stars in the Supplement to the Yale Bright Star Catalog (Hoffleit et al. 1983), for which no radial velocity was listed in that publication. That program makes use of the coudé spectrograph of the Dominion Astrophysical Observatory's 1.22-m telescope, at a dispersion of $0.24 \mathrm{~nm} \mathrm{~mm}{ }^{-1}$. The three binaries whose orbits are presented here are among the first to be discovered in this program, in part due to their relatively short periods and consequent rapid changes in radial velocity.

\section{BACKGROUND INFORMATION}

\subsection{HD 121212}

HD 121212 (HIP 67803, CW CVn, $\alpha=$ $13^{h} 52^{m} 13^{s}, \delta=33^{\circ} 47^{\prime} 12^{\prime \prime}$ [2000]) is to be found in

\footnotetext{
${ }^{1}$ University of Victoria, BC, Canada.

${ }^{2}$ Guest worker, Dominion Astrophysical Observatory, Herzberg Institute of Astrophysics, National Research Council of Canada.
}

the eastern part of Canes Venatici, following $\alpha \mathrm{CVn}$ by almost exactly an hour at a closely similar declination. Its $B V$ magnitude and colour were determined by Hipparcos and are listed in the Tycho-2 catalogue (Hog et al. 2000) as $V=6.97, B-V=$ 1.49, consistent with its MK type, K5 III.

The Gaia (2018) parallax of HD 121212 is $\pi=$ $2.31 \pm 0.05$ mas, which yields $\mathrm{M}_{v}=-1.21 \pm 0.28$, in good agreement with its classification as a late $\mathrm{K}$ giant. Gaia also measured its proper motion components, which are -41.79 mas $\mathrm{yr}^{-1}$ in right ascension and -3.17 mas $\mathrm{yr}^{-1}$ in declination. Together with the parallax these yield the velocity of $71.5 \mathrm{~km} \mathrm{~s}^{-1}$ perpendicular to the line of sight.

HD 121212 was found by Hipparcos (ESA 1997) to be a semiregular variable star of type SRD. However, its amplitude is $0^{m} .25$, much larger than that of most variables of that type (Adelman 2000). Over the years it did seem to the author to vary in brightness to a modest extent, but this seemed to be uncorrelated with the variation in radial velocity. 
TABLE 1

STANDARD STARS USED FOR CROSS-CORRELATION

\begin{tabular}{|c|c|c|c|c|c|}
\hline Standard & Spectral & $R . V$. & & Binaries & \\
\hline Star & Type & $\mathrm{km} \mathrm{s}^{-1}$ & HD 121212 & HD 148434 & HD 148912 \\
\hline$\alpha$ Ari & K2 III & $14.49 \pm 0.03$ & & $\mathrm{x}$ & $\mathrm{x}$ \\
\hline$\alpha$ Boo & K2 III & $-5.30 \pm 0.03$ & & $\mathrm{x}$ & $\mathrm{x}$ \\
\hline$\alpha$ Cas & K0 II-III & $-4.25 \pm 0.03$ & & $\mathrm{x}$ & $\mathrm{x}$ \\
\hline$\alpha$ Hya & K3 III & $-4.40 \pm 0.06$ & $\mathrm{x}$ & & $\mathrm{x}$ \\
\hline$\alpha$ Tau & K5 III & $54.26 \pm 0.04$ & $\mathrm{x}$ & & $\mathrm{x}$ \\
\hline$\beta \mathrm{Gem}$ & K0 III & $3.28 \pm 0.02$ & & $\mathrm{x}$ & $\mathrm{x}$ \\
\hline$\beta \mathrm{Oph}$ & K2 III & $-12.20 \pm 0.02$ & & $\mathrm{x}$ & $\mathrm{x}$ \\
\hline$\delta \mathrm{Oph}$ & M1 III & $-19.40 \pm 0.07$ & $\mathrm{x}$ & & $\mathrm{x}$ \\
\hline$\mu \mathrm{Psc}$ & K4 III & $34.45 \pm 0.05$ & $\mathrm{x}$ & & $\mathrm{x}$ \\
\hline $16 \mathrm{Vir}$ & K0 III & $36.52 \pm 0.04$ & & $\mathrm{x}$ & $\mathrm{x}$ \\
\hline 35 Peg & K0 III & $54.36 \pm 0.03$ & & $\mathrm{x}$ & $\mathrm{x}$ \\
\hline HR 3145 & K2 III & $71.71 \pm 0.08$ & & $\mathrm{x}$ & $\mathrm{x}$ \\
\hline
\end{tabular}

\section{2. $H D 148434$}

HD 148434 (HIP 80528, $\alpha=16^{h} 26^{m} 22^{s}, \delta=$ $40^{\circ} 48^{\prime} 34^{\prime \prime}$ [2000]) is located in the northern part of the constellation of Hercules, a little to the south of $\sigma$ Her. Its BV magnitude and colour were found by Hipparcos (Hog et al. 2000) to be $V=6.91, B-V=$ 1.11, in agreement with its spectral type of K0 III.

The Gaia (2018) parallax of HD 148434 is $\pi=$ $7.31 \pm 0.03$ mas, which yields $\mathrm{M}_{v}=1.23 \pm 0.01$, supporting its classification as a giant. Gaia also measured the proper motion of HD 148434, obtaining $\mu_{\alpha}=21.368 \pm 0.033$ mas $\mathrm{yr}^{-1}, \mu_{\delta}=-53.462 \pm$ 0.035 mas yr$^{-1}$. With the parallax these yield a tangential velocity of $36.2 \mathrm{~km} \mathrm{~s}^{-1}$.

\subsection{HD 148912}

HD 148912 (HIP 80907, $\alpha=16^{h} 31^{m} 21^{s}, \delta=$ $1^{\circ} 18^{\prime} 32^{\prime \prime}$ [2000]) lies in the constellation of Ophiuchus, but close to its boundaries with both Hercules and Serpens, and near the bright star $\lambda$ Oph. Its BV magnitude and colour are $V=6.78, B-V=1.39$ and its MK type is K2-3 III.

The Gaia (2018) parallax of HD 148912 is $\pi=$ $2.51 \pm 0.11$ mas, which yields $\mathrm{M}_{v}=-1.22 \pm 0.10$, consistent with its status as a late-K giant. The proper motion components, also measured by Gaia,

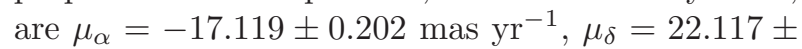
$0.138 \mathrm{mas} \mathrm{yr}^{-1}$. The tangential velocity determined from these and the parallax is $52.8 \mathrm{~km} \mathrm{~s}^{-1}$.

\section{OBSERVATIONS}

Initially the radial velocities were determined at the telescope with the radial-velocity spectrometer (RVS) (Fletcher et al. 1982). Numerous observations of IAU standard stars have been used to adjust the zero-point of the RVS system to the scale defined by Scarfe (2010). Since 2004, when the spectrometer was decommissioned, observations have been made through the same spectrograph optics, but using a CCD as detector, and reduced with a 'pipeline' program developed by D. Bohlender. Radial velocities were determined by averaging the results from crosscorrelation with standard stars. The choice of standard stars for each binary is based on the precision of the cross-correlation, which in turn depends on the match in spectral type between the binary and the standards. The standards for each binary discussed here are listed in Table 1, where the velocities are on the scale and zero-point of Scarfe (2010), although the velocities differ very slightly from those in that paper, owing to the inclusion of slightly more data since its publication. The uncertainties are those of the mean value, and the binaries for which each standard was used are indicated.

For HD 121212, 95 observations were obtained with the RVS between JD 2447216 and 2452880, and 68 with the CCD between JD 2453117 and JD 2457497. The total span of 10281 days is just over 87 orbital cycles, so that the period is determined quite precisely, with a formal uncertainty of less than three minutes. The RVS velocities are presented in Table 2, and those obtained with the CCD in Table 3.

For HD 148434, 42 observations were obtained with the RVS between JDs 2447303 and 2452886, followed by 76 with the CCD between JDs 2453117 and 2457941. The span of 10638 days is just over 50 orbital cycles, and as a result the period of this binary is also determined fairly precisely, with a for- 
THREE SPECTROSCOPIC BINARIES

TABLE 2

RVS RADIAL VELOCITIES OF HD 121212

\begin{tabular}{|c|c|c|c|c|c|c|c|}
\hline $\begin{array}{c}\text { Julian Date } \\
-2,400,000\end{array}$ & $\begin{array}{l}\text { Cycle No. } \\
\& \text { Phase }\end{array}$ & $\begin{array}{c}R . V . \\
\mathrm{km} \mathrm{s}^{-1}\end{array}$ & $\begin{array}{c}O-C \\
\mathrm{~km} \mathrm{~s}^{-1}\end{array}$ & $\begin{array}{c}\text { Julian Date } \\
-2,400,000\end{array}$ & $\begin{array}{l}\text { Cycle No. } \\
\text { \& Phase }\end{array}$ & $\begin{array}{c}R . V . \\
\mathrm{km} \mathrm{s}^{-1}\end{array}$ & $\begin{array}{c}O-C \\
\mathrm{~km} \mathrm{~s}^{-1}\end{array}$ \\
\hline 47216.01 & 0.0553 & -22.7 & -0.6 & 50973.80 & 31.8653 & 5.1 & $-3.0^{\mathrm{a}}$ \\
\hline 47259.88 & .4267 & -18.5 & 1.0 & 50976.74 & .8903 & 5.0 & 0.0 \\
\hline 47263.87 & .4605 & -13.7 & 0.7 & 51166.13 & 33.4935 & -9.3 & -0.1 \\
\hline 47291.79 & .6968 & 12.7 & -1.8 & 51224.04 & .9836 & -10.9 & -0.8 \\
\hline 47303.77 & .7982 & 14.0 & -0.1 & 51279.88 & 34.4564 & -15.4 & -0.4 \\
\hline 47324.72 & .9755 & -8.8 & -0.1 & 51284.86 & .4985 & -8.8 & -0.4 \\
\hline 47346.77 & 1.1622 & -33.9 & 0.5 & 51309.86 & .7101 & 15.0 & -0.0 \\
\hline 47676.76 & 3.9556 & -4.7 & 0.6 & 51317.80 & .7773 & 13.4 & -1.6 \\
\hline 47694.74 & 4.1078 & -30.0 & -0.6 & 51325.79 & .8450 & 10.2 & -0.2 \\
\hline 47725.76 & .3704 & -26.6 & 0.6 & 51333.74 & .9123 & 1.4 & -0.4 \\
\hline 47940.02 & 6.1841 & -36.4 & -0.6 & 51344.74 & 35.0054 & -15.0 & -1.1 \\
\hline 47962.96 & .3783 & -26.7 & -0.5 & 51365.73 & .1831 & -34.9 & 0.8 \\
\hline 47996.87 & .6654 & 13.6 & 1.0 & 51370.73 & .2254 & -37.0 & -0.3 \\
\hline 48037.76 & 7.0115 & -15.0 & -0.2 & 51623.93 & 37.3688 & -27.2 & 0.1 \\
\hline 48059.76 & .1978 & -36.1 & 0.2 & 51634.88 & .4614 & -15.2 & -1.0 \\
\hline 48075.72 & .3328 & -30.6 & 0.7 & 51662.86 & .6984 & 14.6 & 0.0 \\
\hline 48080.72 & .3752 & -26.2 & 0.3 & 51689.79 & .9263 & -0.2 & 0.3 \\
\hline 48388.80 & 9.9831 & -10.8 & -0.8 & 51697.72 & .9934 & -11.4 & 0.3 \\
\hline 48418.73 & 10.2365 & -36.5 & 0.2 & 51711.76 & 38.1123 & -30.2 & -0.4 \\
\hline 48605.06 & 11.8138 & 13.6 & 0.5 & 51718.74 & .1714 & -35.2 & -0.1 \\
\hline 48681.97 & 12.4648 & -14.4 & -0.7 & 51730.77 & .2732 & -33.8 & 1.8 \\
\hline 48730.81 & .8782 & 7.5 & 0.9 & 51737.72 & .3320 & -31.1 & 0.3 \\
\hline 48735.84 & .9208 & 0.5 & 0.0 & 51785.67 & .7379 & 15.3 & -0.3 \\
\hline 48780.75 & 13.3010 & -34.3 & -0.3 & 52009.89 & 40.6360 & 9.8 & -0.2 \\
\hline 48960.05 & 14.8188 & 13.0 & 0.3 & 52026.84 & .7794 & 14.6 & -0.4 \\
\hline 48998.08 & 15.1407 & -32.0 & 0.7 & 52033.79 & .8383 & 11.3 & 0.3 \\
\hline 49041.96 & .5122 & -6.1 & 0.2 & 52044.80 & .9315 & -0.2 & 1.1 \\
\hline 49061.07 & .6739 & 13.2 & 0.0 & 52052.76 & .9988 & -11.9 & 0.8 \\
\hline 49084.90 & .8756 & 6.6 & -0.2 & 52073.74 & 41.1765 & -34.7 & 0.6 \\
\hline 49121.84 & 16.1883 & -36.2 & -0.3 & 52086.74 & .2865 & -34.5 & 0.5 \\
\hline 49370.08 & 18.2897 & -34.2 & 0.5 & 52096.73 & .3711 & -27.7 & -0.6 \\
\hline 49417.97 & .6951 & 14.8 & 0.3 & 52150.67 & .8277 & 11.0 & -1.0 \\
\hline 49442.93 & .9064 & 3.8 & 1.2 & 52387.85 & 43.8355 & 11.2 & -0.1 \\
\hline 49459.84 & 19.0496 & -22.0 & -0.8 & 52417.79 & 44.0889 & -26.5 & 0.4 \\
\hline 49507.75 & .4551 & -15.7 & -0.5 & 52438.74 & .2662 & -35.5 & 0.5 \\
\hline 49557.71 & .8780 & 7.2 & 0.7 & 52460.72 & .4523 & -15.2 & 0.4 \\
\hline 49803.91 & 21.9621 & -6.2 & 0.2 & 52465.73 & .4947 & -8.8 & 0.3 \\
\hline 49849.80 & 22.3506 & -28.8 & 0.6 & 52481.71 & .6300 & 10.0 & 0.5 \\
\hline 50125.04 & 24.6805 & 13.3 & -0.4 & 52648.11 & 46.0385 & -18.2 & 1.2 \\
\hline 50160.95 & .9845 & -10.0 & 0.2 & 52702.98 & .5031 & -8.0 & -0.2 \\
\hline 50181.89 & 25.1618 & -33.2 & 1.2 & 52761.84 & 47.0013 & -14.1 & -1.0 \\
\hline 50232.76 & .5924 & 4.4 & -0.8 & 52768.83 & .0604 & -23.9 & -1.1 \\
\hline 50279.71 & .9898 & -13.3 & $-2.2^{\mathrm{a}}$ & 52774.78 & .1108 & -30.2 & -0.5 \\
\hline 50504.00 & 27.8884 & 7.6 & $2.4^{\mathrm{a}}$ & 52796.78 & .2971 & -36.1 & -1.9 \\
\hline 50577.79 & 28.5131 & -5.9 & 0.3 & 52809.73 & .4067 & -22.8 & -0.5 \\
\hline 50917.93 & 31.3925 & -26.0 & -0.7 & 52817.72 & .4744 & -12.5 & -0.3 \\
\hline 50925.88 & .4597 & -14.4 & 0.1 & 52880.66 & 48.0071 & -13.4 & 0.8 \\
\hline 50931.83 & .5100 & -7.3 & -0.7 & & & & \\
\hline
\end{tabular}

${ }^{\text {a }}$ Rejected observation.

mal uncertainty of a little more than an hour. The RVS data are presented in Table 4 and those from the CCD in Table 5.
For HD 148912, 68 observations were obtained with the RVS between JDs 2447263 and 2452886, followed by 82 with the CCD between JDs 2453117 
TABLE 3

CCD RADIAL VELOCITIES OF HD 121212

\begin{tabular}{|c|c|c|c|c|c|c|c|}
\hline $\begin{array}{l}\text { Julian Date } \\
-2,400,000\end{array}$ & $\begin{array}{l}\text { Cycle No. } \\
\& \text { Phase }\end{array}$ & $\begin{array}{c}R . V . \\
\mathrm{km} \mathrm{s}^{-1}\end{array}$ & $\begin{array}{c}O-C \\
\mathrm{~km} \mathrm{~s}^{-1}\end{array}$ & $\begin{array}{c}\text { Julian Date } \\
-2,400,000\end{array}$ & $\begin{array}{l}\text { Cycle No. } \\
\text { \& Phase }\end{array}$ & $\begin{array}{c}R . V . \\
\mathrm{km} \mathrm{s}^{-1}\end{array}$ & $\begin{array}{l}O-C \\
\mathrm{~km} \mathrm{~s}^{-1}\end{array}$ \\
\hline 53117.84 & 50.0149 & -15.93 & -0.47 & 54531.97 & 61.9857 & -10.26 & 0.17 \\
\hline 53132.81 & .1416 & -33.12 & -0.27 & 54561.91 & 62.2391 & -36.57 & 0.07 \\
\hline 53144.79 & .2430 & -36.43 & 0.16 & 54585.86 & .4418 & -17.31 & -0.10 \\
\hline 53165.72 & .4202 & -20.72 & -0.29 & 54593.81 & .5091 & -6.64 & 0.14 \\
\hline 53180.73 & .5473 & -0.96 & 0.06 & 54594.81 & .5176 & -5.07 & 0.40 \\
\hline 53187.73 & .6065 & 6.99 & 0.12 & 54602.77 & .5850 & 4.53 & 0.33 \\
\hline 53204.74 & .7505 & 15.54 & -0.05 & 54614.76 & .6864 & 13.57 & -0.40 \\
\hline 53213.76 & .8264 & 11.82 & -0.28 & 54625.75 & .7795 & 14.92 & -0.03 \\
\hline 53418.04 & 52.5561 & 0.40 & 0.15 & 54853.10 & 64.7040 & 14.72 & -0.10 \\
\hline 53425.01 & .6151 & 8.24 & 0.38 & 54926.90 & 65.3287 & -31.84 & -0.14 \\
\hline 53452.94 & .8515 & 9.29 & -0.40 & 54929.90 & .3541 & -29.60 & -0.53 \\
\hline 53468.96 & .9871 & -10.40 & 0.28 & 54966.81 & .6666 & 13.01 & 0.32 \\
\hline 53495.74 & 53.2138 & -36.21 & 0.40 & 54987.74 & .8438 & 10.80 & 0.30 \\
\hline 53507.78 & .3158 & -33.37 & -0.51 & 55013.73 & 66.0638 & -23.37 & -0.01 \\
\hline 53515.78 & .3835 & -25.85 & -0.35 & 55302.90 & 68.5116 & -6.61 & -0.22 \\
\hline 53525.74 & .4678 & -13.37 & -0.16 & 55310.87 & .5791 & 3.88 & 0.46 \\
\hline 53542.74 & .6117 & 7.32 & -0.15 & 55321.82 & .6718 & 13.03 & -0.03 \\
\hline 53594.69 & 54.0515 & -21.75 & -0.28 & 55671.89 & 71.6351 & 10.38 & 0.41 \\
\hline 53782.97 & 55.6453 & 11.07 & 0.14 & 56038.87 & 74.7416 & 15.72 & 0.11 \\
\hline 53785.03 & .6627 & 12.63 & 0.23 & 56391.90 & 77.7301 & 15.06 & -0.46 \\
\hline 53788.96 & .6961 & 14.32 & -0.15 & 56405.87 & .8483 & 10.20 & 0.17 \\
\hline 53823.94 & .9921 & -11.67 & -0.13 & 56419.81 & .9663 & -6.53 & 0.57 \\
\hline 53836.87 & 56.1015 & -28.69 & -0.13 & 56661.09 & 80.0089 & -14.95 & -0.52 \\
\hline 53850.86 & .2199 & -36.56 & 0.12 & 56661.95 & .0161 & -16.39 & -0.73 \\
\hline 53860.80 & .3041 & -33.89 & -0.11 & 56663.95 & .0330 & -18.56 & -0.06 \\
\hline 53871.81 & .3973 & -23.77 & -0.11 & 56678.90 & .1596 & -34.28 & 0.00 \\
\hline 53906.78 & .6933 & 14.27 & -0.07 & 56779.87 & 81.0143 & -14.45 & 0.91 \\
\hline 53916.75 & .7777 & 14.52 & -0.50 & 57089.96 & 83.6393 & 10.62 & 0.24 \\
\hline 53927.74 & .8707 & 7.90 & 0.43 & 57098.97 & .7155 & 15.04 & -0.17 \\
\hline 54180.96 & 59.0143 & -15.74 & -0.38 & 57119.91 & .8928 & 5.01 & 0.44 \\
\hline 54238.78 & .5037 & -7.62 & -0.01 & 57151.80 & 84.1627 & -34.51 & -0.01 \\
\hline 54245.79 & .5631 & 1.29 & 0.05 & 57167.79 & .2981 & -33.30 & 0.91 \\
\hline 54253.75 & .6305 & 9.34 & -0.17 & 57465.01 & 86.8141 & 12.71 & -0.35 \\
\hline 54285.73 & .9011 & 3.95 & 0.57 & 57496.84 & 87.0835 & -26.30 & -0.10 \\
\hline
\end{tabular}

and 2457941. The span of 10678 days is over 22 orbital cycles, and as a result of this and the relatively high eccentricity, the period of this binary is also determined fairly precisely, with a formal uncertainty of a little less than an hour. The RVS data are presented in Table 6 and those from the CCD in Table 7.

\section{ORBITS}

For all three binaries, solutions were obtained from the RVS and CCD data separately, and weights based upon the standard deviations from those solutions were assigned for a solution from the combined data. The weight for the CCD data was always set to 1.0. In all cases the weight for the RVS data was lower than for the CCD data, being 0.25, 0.18 and 0.10 respectively for HD 121212, HD 148434 and HD 148912. The orbital elements are presented in Table 8 for all three binaries, and their velocity curves are given in Figures 1, 2 and 3.

\section{DISCUSSION}

All three binaries discussed in this paper show no trace of faint companions in our data, and it must be concluded that the companions must be at least 2.5 magnitudes fainter than the primaries, especially if their spectral types are similar, since then they are likely to cause some slight distortion of the cross correlation function. Moreover, if the secondaries are main sequence stars a difference of only 2.5 magnitudes would indicate that the secondaries are of type 
TABLE 4

RVS RADIAL VELOCITIES OF HD 148434

\begin{tabular}{|c|c|c|c|c|c|c|c|}
\hline $\begin{array}{c}\text { Julian Date } \\
-2,400,000\end{array}$ & $\begin{array}{l}\text { Cycle No. } \\
\text { \& Phase }\end{array}$ & $\begin{array}{c}R . V . \\
\mathrm{km} \mathrm{s}^{-1}\end{array}$ & $\begin{array}{c}O-C \\
\mathrm{~km} \mathrm{~s}^{-1}\end{array}$ & $\begin{array}{c}\text { Julian Date } \\
-2,400,000\end{array}$ & $\begin{array}{l}\text { Cycle No. } \\
\text { \& Phase }\end{array}$ & $\begin{array}{c}R . V . \\
\mathrm{km} \mathrm{s}^{-1}\end{array}$ & $\begin{array}{l}O-C \\
\mathrm{~km} \mathrm{~s}^{-1}\end{array}$ \\
\hline 47303.87 & 0.5629 & -4.4 & -0.2 & 52184.63 & .4274 & -5.8 & 1.1 \\
\hline 47324.83 & 0.6610 & -2.6 & -0.3 & 52387.93 & 24.3797 & -8.1 & -0.5 \\
\hline 47328.81 & 0.6797 & -2.0 & -0.1 & 52417.89 & .5201 & -5.1 & 0.0 \\
\hline 49508.84 & 10.8923 & -1.2 & 0.1 & 52438.84 & .6182 & -2.8 & 0.3 \\
\hline 50973.86 & 17.7553 & -1.3 & -0.3 & 52460.77 & .7210 & -1.0 & 0.3 \\
\hline 51689.86 & 21.1096 & -6.5 & 0.1 & 52465.80 & .7445 & -1.1 & -0.1 \\
\hline 51697.78 & .1467 & -6.5 & 0.8 & 52481.76 & .8193 & -1.0 & -0.3 \\
\hline 51718.80 & .2451 & -8.4 & 0.1 & 52488.72 & .8519 & -0.7 & 0.2 \\
\hline 51730.77 & .3012 & -8.3 & 0.0 & 52516.69 & .9830 & -3.9 & -0.7 \\
\hline 51785.69 & .5585 & -5.0 & -0.7 & 52537.66 & 25.0812 & -4.7 & 1.2 \\
\hline 51796.69 & .6100 & -3.4 & -0.2 & 52544.64 & .1139 & -7.1 & -0.4 \\
\hline 51824.63 & .7409 & -0.9 & 0.2 & 52564.63 & .2075 & -8.0 & 0.2 \\
\hline 51952.06 & 22.3379 & -7.6 & 0.6 & 52579.60 & .2776 & -8.9 & -0.4 \\
\hline 52010.96 & .6138 & -2.7 & 0.4 & 52648.12 & .5986 & -2.6 & 0.9 \\
\hline 52026.93 & .6886 & -1.6 & 0.2 & 52761.95 & 26.1319 & -8.2 & -1.2 \\
\hline 52051.88 & .8055 & -0.8 & -0.2 & 52774.85 & .1923 & -8.1 & -0.1 \\
\hline 52073.83 & .9083 & -1.7 & -0.1 & 52796.84 & .2953 & -9.7 & -1.4 \\
\hline 52086.81 & .9691 & -2.6 & 0.2 & 52809.82 & .3562 & -6.0 & 1.9 \\
\hline 52096.78 & 23.0158 & -4.0 & 0.1 & 52817.80 & .3935 & -7.2 & 0.2 \\
\hline 52150.66 & .2682 & -8.8 & -0.3 & 52873.74 & .6556 & -2.5 & -0.2 \\
\hline 52163.68 & .3292 & -8.7 & -0.5 & 52885.72 & .7117 & -0.9 & 0.5 \\
\hline
\end{tabular}

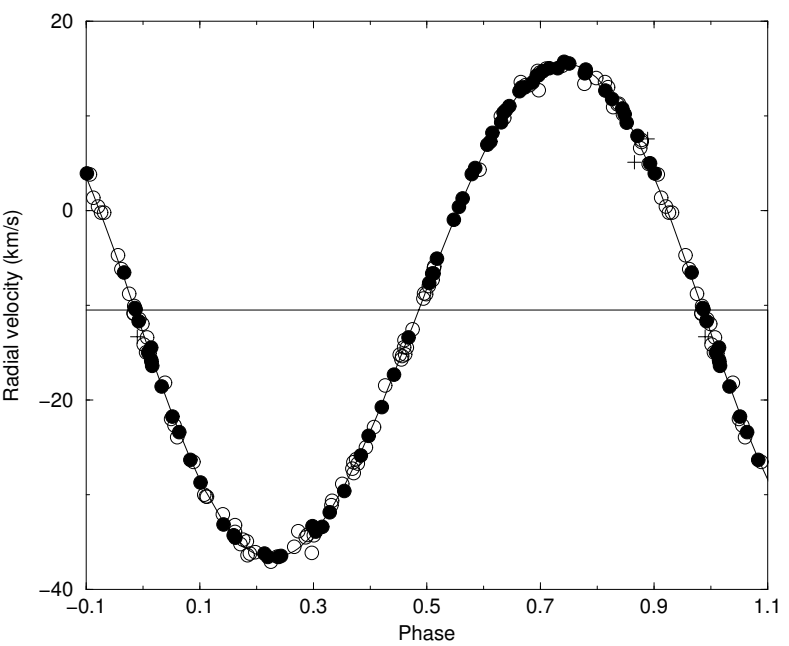

Fig. 1. The observed radial velocities of HD 121212, with the curve derived from the adopted elements drawn through them. The DAO CCD data are shown as filled circles and the spectrometer data as open circles. Rejected RVS velocities are shown as plus signs.

about A0 for HD 121212 and HD 148912, and about F5 for HD 148434. The former would have mass near $3 M_{\odot}$, and the latter, $1.3 M_{\odot}$. Either should be detectable in the system's colour indices, and indeed in the spectra of HD 121212 and 148912. Thus

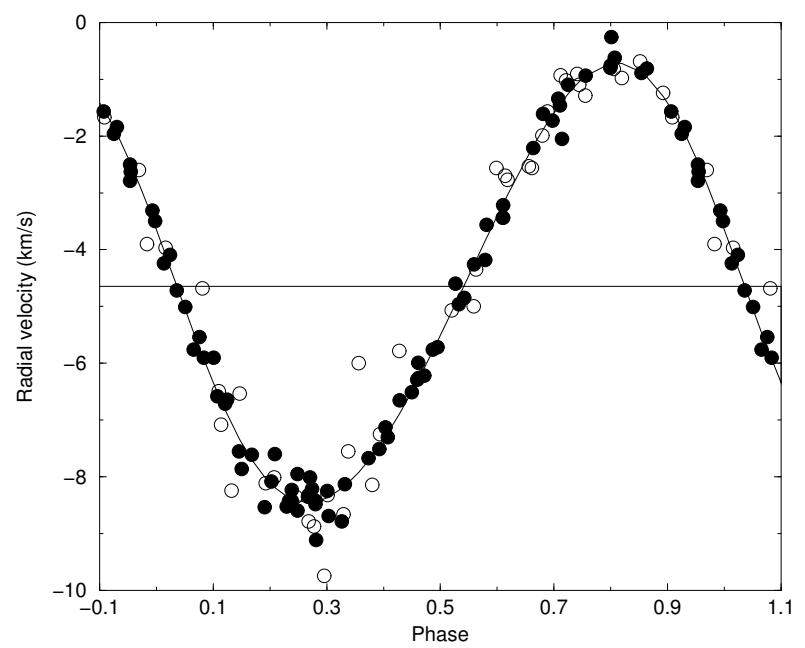

Fig. 2. The observed radial velocities of HD 148434, with the curve derived from the adopted elements drawn through them. The symbols have the same meaning as in Figure 1, except that there are no rejected data.

we conclude that the secondaries are either mainsequence stars that are fainter than the primaries by more than 2,5 magnitudes, or slightly evolved subgiants, in which case their masses are only a little less than those of the primaries, given the evolutionary timescales of stars once they leave the main se- 
TABLE 5

CCD RADIAL VELOCITIES OF HD 148434

\begin{tabular}{|c|c|c|c|c|c|c|c|}
\hline $\begin{array}{c}\text { Julian Date } \\
-2,400,000\end{array}$ & $\begin{array}{c}\text { Cycle No. } \\
\text { \& Phase }\end{array}$ & $\begin{array}{c}R . V . \\
\mathrm{km} \mathrm{s}^{-1}\end{array}$ & $\begin{array}{c}O-C \\
\mathrm{~km} \mathrm{~s}^{-1}\end{array}$ & $\begin{array}{l}\text { Julian Date } \\
-2,400,000\end{array}$ & $\begin{array}{l}\text { Cycle No. } \\
\text { \& Phase }\end{array}$ & $\begin{array}{c}R . V . \\
\mathrm{km} \mathrm{s}^{-1}\end{array}$ & $\begin{array}{c}O-C \\
\mathrm{~km} \mathrm{~s}^{-1}\end{array}$ \\
\hline 53117.97 & 27.7997 & -0.76 & -0.04 & 54695.71 & .1908 & -8.53 & -0.52 \\
\hline 53145.86 & .9304 & -1.84 & 0.12 & 54714.69 & .2797 & -8.42 & 0.01 \\
\hline 53165.86 & 28.0240 & -4.09 & 0.22 & 54724.68 & .3265 & -8.78 & -0.58 \\
\hline 53213.77 & .2485 & -7.95 & 0.47 & 54926.98 & 36.2742 & -8.21 & 0.23 \\
\hline 53258.71 & .4590 & -6.29 & 0.03 & 54966.94 & .4614 & -6.26 & 0.02 \\
\hline 53425.09 & 29.2384 & -8.23 & 0.15 & 54987.87 & .5595 & -4.26 & -0.00 \\
\hline 53515.86 & .6637 & -2.21 & -0.03 & 54998.80 & .6107 & -3.44 & -0.25 \\
\hline 53525.82 & .7103 & -1.46 & -0.02 & 55013.77 & .6808 & -1.61 & 0.28 \\
\hline 53544.78 & .7991 & -0.80 & -0.08 & 55050.72 & .8539 & -0.89 & -0.01 \\
\hline 53601.68 & 30.0657 & -5.76 & -0.30 & 55302.95 & 38.0355 & -4.72 & -0.09 \\
\hline 53618.71 & .1455 & -7.55 & -0.23 & 55321.94 & .1245 & -6.64 & 0.26 \\
\hline 53619.69 & .1501 & -7.86 & -0.46 & 55386.73 & .4280 & -6.65 & 0.23 \\
\hline 53640.65 & .2482 & -8.59 & -0.17 & 55393.78 & .4610 & -5.99 & 0.29 \\
\hline 53647.63 & .2810 & -9.11 & -0.68 & 55407.76 & .5265 & -4.60 & 0.36 \\
\hline 53785.06 & .9248 & -1.96 & -0.11 & 55425.71 & .6106 & -3.22 & -0.03 \\
\hline 53823.99 & 31.1071 & -6.58 & -0.07 & 55720.80 & 39.9929 & -3.31 & 0.15 \\
\hline 53836.96 & .1679 & -7.61 & 0.09 & 55766.76 & 40.2083 & -7.60 & 0.59 \\
\hline 53850.98 & .2336 & -8.42 & -0.06 & 56106.75 & 41.8010 & -0.26 & 0.46 \\
\hline 53860.92 & .2801 & -8.48 & -0.05 & 56148.74 & .9977 & -3.50 & 0.08 \\
\hline 53871.90 & .3316 & -8.13 & 0.03 & 56170.71 & 42.1006 & -5.90 & 0.46 \\
\hline 53906.83 & .4952 & -5.72 & -0.11 & 56405.93 & 43.2025 & -8.08 & 0.05 \\
\hline 53914.79 & .5325 & -4.96 & -0.13 & 56419.92 & .2681 & -8.32 & 0.12 \\
\hline 53916.83 & .5420 & -4.85 & -0.22 & 56486.77 & .5813 & -3.56 & 0.24 \\
\hline 53994.69 & .9068 & -1.57 & -0.05 & 56513.73 & .7075 & -1.34 & 0.14 \\
\hline 54004.67 & .9536 & -2.79 & -0.33 & 56779.99 & 44.9549 & -2.63 & -0.14 \\
\hline 54238.84 & 33.0506 & -5.01 & 0.04 & 56853.76 & 45.3005 & -8.25 & 0.11 \\
\hline 54245.84 & .0834 & -5.90 & 0.03 & 56875.73 & .4034 & -7.13 & 0.16 \\
\hline 54253.85 & .1209 & -6.71 & 0.11 & 57090.04 & 46.4073 & -7.30 & -0.08 \\
\hline 54285.75 & .2703 & -8.01 & 0.43 & 57099.01 & .4494 & -6.51 & -0.01 \\
\hline 54292.75 & .3031 & -8.69 & -0.34 & 57151.92 & .6972 & -1.73 & -0.10 \\
\hline 54307.72 & .3732 & -7.67 & 0.04 & 57232.74 & 47.0758 & -5.54 & 0.19 \\
\hline 54328.71 & .4716 & -6.22 & -0.14 & 57478.99 & 48.2294 & -8.52 & -0.18 \\
\hline 54351.67 & .5791 & -4.18 & -0.34 & 57486.99 & .2669 & -8.35 & 0.09 \\
\hline 54593.91 & 34.7139 & -2.05 & -0.65 & 57533.87 & .4865 & -5.76 & 0.02 \\
\hline 54602.91 & .7561 & -0.94 & 0.00 & 57584.75 & .7249 & -1.10 & 0.16 \\
\hline 54613.84 & .8073 & -0.62 & 0.10 & 57847.00 & 49.9534 & -2.50 & -0.04 \\
\hline 54625.85 & .8635 & -0.81 & 0.15 & 57907.82 & 50.2384 & -8.42 & -0.04 \\
\hline 54657.79 & 35.0132 & -4.24 & -0.23 & 57940.75 & .3926 & -7.51 & -0.06 \\
\hline
\end{tabular}

quence. One final possible alternative is that the secondaries are white dwarfs, which would again be very much fainter than the primaries, offering little hope of detection.

Despite these considerations, it might be possible to detect the companions interferometrically. The best chance of doing so, as suggested in the preceding paragraph, is in the case that they are evolved objects only slightly less massive than the primaries. We estimate below values of the angular size of the major semiaxes of the systems' orbits. As an example, we make the plausible assumption that the primary star's mass is twice that of the sun for all three systems. We make use of the following two equations. The first is the equation of the mass function, in which $M_{1}$ is the primary's mass, $M_{2}$ is that of the secondary, and $q=M_{2} / M_{1}$.

$$
\frac{f(M)}{M_{1} \sin ^{3} i}=\frac{q^{3}}{(1+q)^{2}}
$$

The second equation is for the projected angular size $a$ of the major semiaxis, in terms of the parallax $\pi$, the mass ratio $q$, the inclination $i$, and the product 
THREE SPECTROSCOPIC BINARIES

TABLE 6

RVS RADIAL VELOCITIES OF HD 148912

\begin{tabular}{|c|c|c|c|c|c|c|c|}
\hline $\begin{array}{c}\text { Julian Date } \\
-2,400,000\end{array}$ & $\begin{array}{c}\text { Cycle No. } \\
\text { \& Phase }\end{array}$ & $\begin{array}{c}R . V . \\
\mathrm{km} \mathrm{s}^{-1}\end{array}$ & $\begin{array}{c}O-C \\
\mathrm{~km} \mathrm{~s}^{-1}\end{array}$ & $\begin{array}{c}\text { Julian Date } \\
-2,400,000\end{array}$ & $\begin{array}{c}\text { Cycle No. } \\
\& \text { Phase }\end{array}$ & $\begin{array}{c}R . V . \\
\mathrm{km} \mathrm{s}^{-1}\end{array}$ & $\begin{array}{c}O-C \\
\mathrm{~km} \mathrm{~s}^{-1}\end{array}$ \\
\hline 47263.97 & 0.6578 & -68.2 & 0.7 & 51796.68 & .0677 & -70.7 & 0.0 \\
\hline 48418.82 & 3.0553 & -69.2 & -0.3 & 51824.61 & .1257 & -75.1 & 0.4 \\
\hline 50232.83 & 6.8211 & -61.9 & -0.0 & 51952.07 & .3903 & -75.4 & -0.3 \\
\hline 50246.83 & .8502 & -60.9 & -0.9 & 52010.97 & .5125 & -72.6 & 0.1 \\
\hline 50518.05 & 7.4132 & -74.7 & 0.0 & 52026.94 & .5457 & -72.2 & -0.2 \\
\hline 50577.89 & .5375 & -72.7 & -0.5 & 52051.89 & .5975 & -70.5 & 0.2 \\
\hline 50609.85 & .6038 & -71.2 & -0.7 & 52073.84 & .6430 & -68.9 & 0.5 \\
\hline 50917.98 & 8.2435 & -77.1 & -0.2 & 52086.82 & .6700 & -68.9 & -0.4 \\
\hline 50925.96 & .2601 & -76.4 & 0.4 & 52096.78 & .6907 & -67.9 & -0.0 \\
\hline 50973.86 & .3595 & -76.2 & -0.6 & 52150.65 & .8025 & -63.5 & -0.6 \\
\hline 51070.64 & .5604 & -72.0 & -0.4 & 52163.69 & .8296 & -61.8 & -0.4 \\
\hline 51279.96 & .9950 & -56.5 & -0.3 & 52184.64 & .8731 & -58.4 & 0.0 \\
\hline 51289.93 & 9.0157 & -60.8 & -0.3 & 52387.94 & 11.2951 & -78.1 & -1.7 \\
\hline 51309.92 & .0572 & -68.8 & 0.4 & 52417.91 & .3573 & -75.0 & 0.6 \\
\hline 51317.93 & .0738 & -72.8 & -1.2 & 52438.85 & .4008 & -74.8 & 0.2 \\
\hline 51325.94 & .0904 & -74.4 & -1.1 & 52460.77 & .4463 & -73.8 & 0.3 \\
\hline 51333.85 & .1068 & -75.6 & -1.1 & 52465.81 & .4568 & -74.0 & -0.1 \\
\hline 51344.80 & .1296 & -76.5 & -0.7 & 52481.77 & .4899 & -73.2 & 0.1 \\
\hline 51365.76 & .1731 & -76.5 & 0.2 & 52488.73 & .5043 & -72.2 & 0.8 \\
\hline 51414.68 & .2746 & -77.4 & -0.7 & 52516.68 & .5624 & -72.1 & -0.5 \\
\hline 51438.64 & .3244 & -76.8 & -0.7 & 52527.67 & .5852 & -71.4 & -0.4 \\
\hline 51444.65 & .3369 & -76.9 & -0.9 & 52544.63 & .6204 & -71.3 & -1.3 \\
\hline 51452.63 & .3534 & -76.1 & -0.4 & 52564.62 & .6619 & -68.5 & 0.3 \\
\hline 51624.00 & .7092 & -67.2 & -0.0 & 52579.59 & .6930 & -67.7 & -0.0 \\
\hline 51632.01 & .7258 & -66.0 & 0.5 & 52648.13 & .8353 & -60.4 & 0.6 \\
\hline 51662.98 & .7901 & -63.7 & -0.1 & 52703.06 & .9493 & -52.8 & 0.6 \\
\hline 51689.86 & .8459 & -60.4 & -0.1 & 52761.94 & 12.0715 & -71.5 & -0.3 \\
\hline 51697.82 & .8624 & -59.4 & -0.3 & 52768.93 & .0860 & -73.2 & -0.3 \\
\hline 51710.80 & .8894 & -57.4 & -0.2 & 52774.86 & .0984 & -73.5 & 0.5 \\
\hline 51718.81 & .9060 & -55.9 & 0.1 & 52796.86 & .1440 & -77.6 & -1.4 \\
\hline 51730.78 & .9309 & -53.5 & 0.7 & 52808.80 & .1688 & -75.2 & 1.5 \\
\hline 51737.75 & .9453 & -53.6 & -0.2 & 52817.80 & .1875 & -77.2 & -0.3 \\
\hline 51785.70 & 10.0449 & -66.5 & 0.4 & 52873.73 & .3036 & -75.5 & 0.9 \\
\hline 51787.70 & .0490 & -67.5 & 0.2 & 52885.72 & .3285 & -75.6 & 0.4 \\
\hline
\end{tabular}

$a_{1} \sin i$, determined from the orbit.

$$
a=\pi\left(a_{1} \sin i\right)\left(1+q^{-1}\right) \csc i .
$$

As indicated above we assume that despite the luminosity differences between the stars, they are similar in mass and surface temperature, as was found for the components of HR 6046 by Scarfe et al. (2007), where the companion was detected with difficulty, despite being almost equal in mass to the primary. Here, let us assume that $q=0.9$ for all three binaries. Then we solve equation 1 for the inclination of each orbit, and insert its cosecant into equation 2 along with the values of $a_{1} \sin i$ from the orbit solutions, the parallaxes and the assumed values of $q$ to find the projected major semiaxes.
An alternative, more direct, approach is to estimate the angular sizes of the systems' major semiaxes, using Kepler's Third Law and their parallaxes, by means of equation (3), in which $a$ is the angular major semiaxis, $\pi$ is the parallax, $P$ is the period in years and $M$ is the total mass of the system in solar units.

$$
a=\pi P^{2 / 3} M^{1 / 3}
$$

A note of caution - choice of too small a value for $M$, coupled with too low a value of the mass ratio $q$, may lead to values of $\sin i$ that exceed unity when the mass function is large, as it is for HD 121212. For example our choice of 3.8 solar masses for the total mass of that system does not permit the mass 
TABLE 7

CCD RADIAL VELOCITIES OF HD 148912

\begin{tabular}{|c|c|c|c|c|c|c|c|}
\hline $\begin{array}{c}\text { Julian Date } \\
-2,400,000\end{array}$ & $\begin{array}{l}\text { Cycle No. } \\
\text { \& Phase }\end{array}$ & $\begin{array}{l}R . V . \\
\mathrm{km} \mathrm{s}^{-1}\end{array}$ & $\begin{array}{l}O-C \\
\mathrm{~km} \mathrm{~s}^{-1}\end{array}$ & $\begin{array}{l}\text { Julian Date } \\
-2,400,000\end{array}$ & $\begin{array}{l}\text { Cycle No. } \\
\text { \& Phase }\end{array}$ & $\begin{array}{l}R . V . \\
\mathrm{km} \mathrm{s}^{-1}\end{array}$ & $\begin{array}{l}O-C \\
\mathrm{~km} \mathrm{~s}^{-1}\end{array}$ \\
\hline 53117.99 & 12.8107 & -62.48 & -0.02 & 54602.89 & .8933 & -56.99 & -0.11 \\
\hline 53144.92 & .8666 & -58.92 & -0.05 & 54625.82 & .9409 & -53.66 & -0.02 \\
\hline 53165.84 & .9100 & -55.39 & 0.22 & 54657.76 & 16.0072 & -58.77 & -0.11 \\
\hline 53180.82 & .9411 & -53.60 & 0.03 & 54695.70 & .0860 & -73.44 & -0.55 \\
\hline 53187.79 & .9556 & -53.23 & 0.00 & 54714.67 & .1254 & -75.37 & 0.17 \\
\hline 53204.78 & .9909 & -55.92 & -0.31 & 54724.66 & .1461 & -76.78 & -0.53 \\
\hline 53213.76 & 13.0095 & -58.87 & 0.28 & 54927.00 & .5662 & -71.35 & 0.15 \\
\hline 53251.68 & .0882 & -73.20 & -0.10 & 54943.99 & .6014 & -70.47 & 0.11 \\
\hline 53258.69 & .1028 & -73.84 & 0.44 & 54966.92 & .6490 & -68.98 & 0.21 \\
\hline 53272.65 & .1318 & -75.80 & -0.01 & 54987.83 & .6925 & -67.67 & 0.08 \\
\hline 53283.61 & .1545 & -76.14 & 0.31 & 54998.80 & .7152 & -67.19 & -0.28 \\
\hline 53418.10 & .4337 & -74.52 & -0.16 & 55013.76 & .7463 & -65.44 & 0.20 \\
\hline 53453.05 & .5063 & -73.16 & -0.26 & 55055.70 & .8333 & -61.03 & 0.08 \\
\hline 53515.86 & .6366 & -69.80 & -0.23 & 55302.99 & 17.3467 & -75.93 & -0.12 \\
\hline 53525.85 & .6574 & -68.82 & 0.11 & 55321.96 & .3861 & -75.08 & 0.12 \\
\hline 53542.79 & .6926 & -67.99 & -0.25 & 55386.75 & .5206 & -72.13 & 0.45 \\
\hline 53545.81 & .6988 & -67.77 & -0.25 & 55393.74 & .5351 & -72.15 & 0.10 \\
\hline 53594.72 & .8004 & -63.02 & 0.01 & 55407.73 & .5641 & -71.46 & 0.09 \\
\hline 53601.69 & .8148 & -62.17 & 0.05 & 55720.84 & 18.2142 & -76.78 & 0.21 \\
\hline 53618.67 & .8501 & -60.15 & -0.13 & 55766.73 & .3094 & -75.73 & 0.58 \\
\hline 53619.68 & .8522 & -60.02 & -0.14 & 56106.79 & 19.0154 & -60.22 & 0.24 \\
\hline 53640.62 & .8957 & -56.55 & 0.15 & 56148.72 & .1024 & -74.00 & 0.26 \\
\hline 53647.61 & .9102 & -55.81 & -0.21 & 56170.69 & .1480 & -76.05 & 0.25 \\
\hline 53785.09 & 14.1956 & -77.05 & -0.10 & 56405.95 & .6364 & -69.71 & -0.13 \\
\hline 53824.02 & .2764 & -76.78 & -0.11 & 56419.95 & .6655 & -68.51 & 0.16 \\
\hline 53836.98 & .3033 & -76.56 & -0.18 & 56486.75 & .8042 & -62.52 & 0.30 \\
\hline 53850.96 & .3323 & -76.12 & -0.11 & 56549.67 & .9348 & -53.78 & 0.17 \\
\hline 53860.94 & .3530 & -75.74 & -0.02 & 56799.87 & 20.4542 & -73.90 & 0.07 \\
\hline 53871.92 & .3758 & -75.37 & -0.01 & 56853.75 & .5661 & -71.56 & -0.06 \\
\hline 53906.86 & .4484 & -74.13 & -0.05 & 56875.71 & .6117 & -70.09 & 0.20 \\
\hline 53916.81 & .4690 & -73.77 & -0.09 & 57090.05 & 21.0566 & -69.01 & 0.06 \\
\hline 53994.65 & .6306 & -69.75 & -0.00 & 57120.02 & .1188 & -75.17 & 0.06 \\
\hline 54004.64 & .6513 & -69.13 & -0.01 & 57160.92 & .2038 & -77.12 & -0.14 \\
\hline 54238.86 & 15.1376 & -75.84 & 0.16 & 57232.73 & .3528 & -75.70 & 0.02 \\
\hline 54245.86 & .1521 & -76.47 & -0.07 & 57479.01 & .8641 & -59.13 & -0.08 \\
\hline 54263.87 & .1895 & -76.83 & 0.09 & 57487.01 & .8807 & -57.73 & 0.11 \\
\hline 54285.77 & .2350 & -76.88 & 0.07 & 57533.89 & .9780 & -54.18 & -0.11 \\
\hline 54292.77 & .2495 & -77.05 & -0.18 & 57584.76 & 22.0836 & -72.72 & -0.07 \\
\hline 54307.75 & .2806 & -76.77 & -0.14 & 57847.02 & .6281 & -69.59 & 0.23 \\
\hline 54328.74 & .3242 & -76.09 & 0.03 & 57907.85 & .7544 & -65.57 & -0.28 \\
\hline 54351.66 & .3718 & -76.30 & $-0.87^{\mathrm{a}}$ & 57940.76 & .8227 & -61.73 & 0.03 \\
\hline
\end{tabular}

${ }^{\text {a }}$ Rejected observation.

ratio to be as small as 0.6. One can avoid this pitfall by checking with equation 1 .

Since our choice of primary mass and system mass ratio are the same for both the method using the mass function and that using Kepler's Third Law, it is not surprising that both methods yield the same results for the angular major semiaxes, 1.7 mas for HD 121212, 9.1 mas for HD 148434, and 5.4 mas for HD 148912. The angular separations at any or- bital phase should be of this order of magnitude for our representative choice of masses, and any plausible masses and mass ratios are unlikely to yield very different results, as indicated by the weak dependence of equation 3 on the total mass. High inclinations like that of HD 121212 will make the separation significantly smaller at times, and large eccentricities like that of HD 148912 will give occasionally larger separations. The major axes estimated here 
THREE SPECTROSCOPIC BINARIES

TABLE 8

TABLE 8 ORBITAL ELEMENTS

\begin{tabular}{lccc}
\hline \multicolumn{1}{c}{ Object } & HD 121212 & HD 148434 & HD 148912 \\
\hline$P($ days $)$ & $118.132 \pm 0.002$ & $213.464 \pm 0.046$ & $481.699 \pm 0.040$ \\
$T(\mathrm{~J} . \mathrm{D}-2,450,000)$ & $3470.5 \pm 1.4$ & $4868.4 \pm 4.8$ & $3690.9 \pm 3.1$ \\
$K\left(\mathrm{~km} \mathrm{~s}^{-1}\right)$ & $26.16 \pm 0.05$ & $3.86 \pm 0.04$ & $11.88 \pm 0.04$ \\
$e$ & $0.026 \pm 0.002$ & $0.070 \pm 0.011$ & $0.497 \pm 0.002$ \\
$\omega($ degrees $)$ & $94.2 \pm 4.3$ & $76.1 \pm 8.1$ & $48.2 \pm 0.3$ \\
$\gamma\left(\mathrm{km} \mathrm{s}^{-1}\right)$ & $-10.49 \pm 0.04$ & $-4.64 \pm 0.03$ & $-69.05 \pm 0.02$ \\
& & & 0.253 \\
S.E. (wt. 1$)\left(\mathrm{km} \mathrm{s}^{-1}\right)$ & 0.341 & $11.31 \pm 0.12$ & 0.197 \\
$a_{1} \sin i(\mathrm{Gm})$ & $42.48 \pm 0.09$ & $0.00127 \pm 0.00004$ & $0.0548 \pm 0.28 \pm 0.25$ \\
$f(M)\left(M_{\odot}\right)$ & $0.2194 \pm 0.0013$ & 0.0006 \\
\hline
\end{tabular}

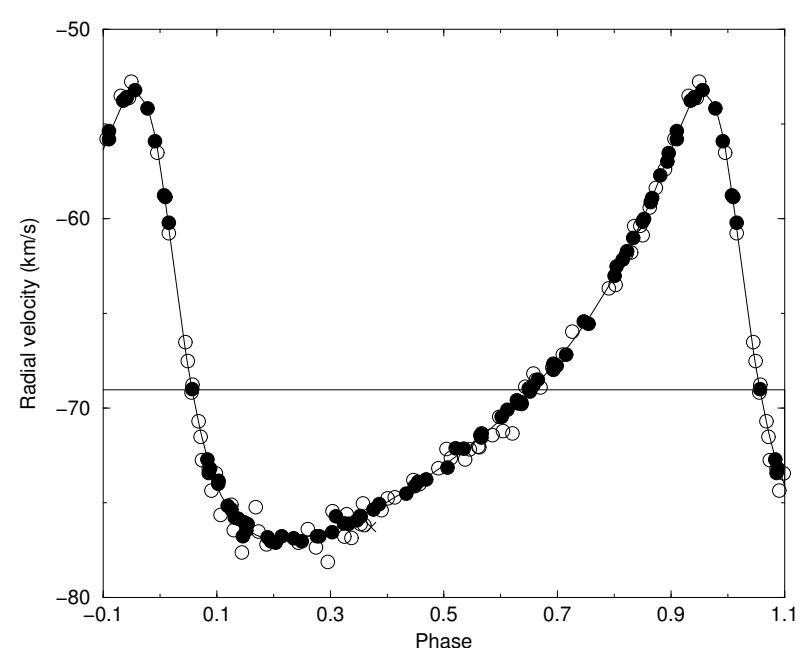

Fig. 3. The observed radial velocities of HR 148912, with the curve derived from the adopted elements drawn through them. The symbols have the same meaning as for Figure 1, except that a rejected CCD velocity is shown as a cross.

would make resolution of the pairs challenging for Gaia and very difficult, if not impossible, for earlier instruments. Nor do any of the inclinations estimated here offer much hope of detecting eclipses, despite the likely angular sizes of the giant primaries. Determination of the actual masses thus remains a challenge.
The author would like to acknowledge the $\mathrm{Na}$ tional Research Council of Canada for the provision of observing time at the Dominion Astrophysical Observatory for a period now exceeding half of the century of that observatory's existence, and especially to thank members of the DAO staff, in particular D. Bohlender, D. Monin and the late L. Saddlemyer for their greatly appreciated support and assistance. He would also like to thank a kindly referee for some helpful advice.

\section{REFERENCES}

Adelman, S. J. 2000, IBVS, 4959, 1

ESA 1997, The Hipparcos and Tycho Catalogs, SP 1200 Fletcher, J. M., Harris, H. C., McClure, R. D., \& Scarfe, C. D. 1982, PASP, 94, 1017

Gaia Collaboration 2018, A\&A, arXiv1804.09365

Hoffleit, D., Saladyga, M. \& Wlasuk, P. 1983, A Supplement to the Bright Star Catalogue (New Haven, CT: Yale Univ. Obs.)

Hog, E., Fabricius, C., Makarov, V. V., et al. 2000, A\&A, $355, \mathrm{~L} 27$

Scarfe, C. D. 2010, Obs, 130, 214

Scarfe, C. D., Griffin, R. F., \& Griffin, R. E. M. 2007, MNRAS, 376, 1671

C. D. Scarfe: Department of Physics and Astronomy, University of Victoria, Victoria, BC V8W 3P6, Canada (scarfe@uvic.ca). 